\title{
USO DE INFORMACIÓN EN DOCENTES UNIVERSITARIOS PERUANOS: UN ANÁLISIS DE CITAS EN TRABAJOS DE INVESTIGACIÓN (2010-2014)
}

\author{
Mónica Arakaki* \\ Pontificia Universidad Católica del Perú (PUCP). Departamento Académico de Humanidades.
}

\begin{abstract}
Resumen: El estudio aplica técnicas de análisis de citas para caracterizar el uso de información de los docentes de cuatro departamentos (Ciencias, Ingeniería, Ciencias Sociales y Humanidades) de la Pontificia Universidad Católica del Perú (PUCP). A partir de la producción académica de este grupo, publicada de 2010 a 2014 e indexada en Scopus, se obtuvo una muestra aleatoria de 217 documentos. De este conjunto, se extrajeron las referencias bibliográficas y se examinaron para determinar su cantidad, edad, idioma y tipo documental. Dadas las diferencias encontradas entre departamentos, se sostiene que el área disciplinar juega un rol importante en la conducta informativa de los académicos.

Palabras clave: Bibliometría; uso de información; análisis de citas; análisis de referencias; estudios de usuarios; PUCP; Perú.

Title: INFORMATION USE BY PERUVIAN FACULTY MEMBERS: A CITATION ANALYSIS OF RESEARCH OUTPUTS (2010-2014).

Abstract: This study applies citation analysis techniques to characterize the information use among faculty members of four departments (Science, Engineering, Social Sciences and Humanities) at the Pontifical Catholic University of Peru (PUCP). A random sample of 217 research outputs was obtained from 2010-2014 faculty publications that were indexed by Scopus. Cited references were gathered and categorized by type and language, and their number and age were computed. Differences were found between departments. Considering the results, it is argued that the discipline itself is a major determinant of the information behavior of academics.

Keywords: Bibliometrics; information use; citation analysis; reference analysis; user studies; PUCP; Peru.
\end{abstract}

Copyright: (C) 2018 Servicio de Publicaciones de la Universidad de Murcia (Spain). Este es un artículo de acceso abierto distribuido bajo los términos de la licencia Creative Commons Reconocimiento 4.0 Internacional (CC BY 4.0).

\section{INTRODUCCIÓN}

Como proceso social, la ciencia se desarrolla a partir de un intercambio continuo de saberes. Conocedoras de su valor, las instituciones destinan recursos para que los investigadores tengan acceso a contribuciones académicas previas. Sin embargo, la sola adquisición y puesta a disposición de resultados de investigaciones no garantiza su aprovechamiento. Hay que conocer las motivaciones, carencias o frustraciones de los investigadores como usuarios cuando interactúan con información. Se requiere determinar cómo caen en la cuenta (o no) de que la necesitan, dónde la buscan, cómo la encuentran, cómo juzgan su relevancia, en qué la usan y, en otros casos, por qué la ignoran o evitan.

El entendimiento de la conducta informativa de los investigadores puede contribuir a diseñar servicios y adquirir recursos en sintonía con sus necesidades y hábitos de uso de información. A su vez, este conocimiento puede tener un impacto positivo en la calidad de los productos derivados de la investigación y en última instancia, de forma agregada, en el desarrollo de una disciplina o sector.

Lo anterior cobra relevancia particular en el Perú pues, después de décadas de reconocer el retraso del país en investigación, se han dado algunas (todavía insuficientes) medidas para impulsarla desde el Gobierno y la academia. Sin embargo, el tema de la conducta informativa de los investigadores no ha sido abordado.

En este sentido, el presente estudio pretende caracterizar el uso de información por parte de docentes investigadores de la Pontificia Universidad Católica del Perú (PUCP), específicamente de cuatro departamentos: Ciencias, Ciencias Sociales, Humanidades e Ingeniería. Para tal fin, se recopiló una muestra de los trabajos publicados por estos académicos entre 2010 y 2014 que se encontraban indizados en Scopus. Se examinaron las referencias

*monica.arakaki@pucp.edu.pe

Recibido: 21-08-2017; $2^{\mathrm{a}}$ versión: 24-02-2018; aceptado: 11-04-2018.

ARAKAKI, M. Uso de información en docentes universitarios peruanos: un análisis de citas en trabajos de investigación (2010-2014). Anales de Documentación, 2018, vol. 21, $\mathrm{n}^{\mathrm{o}} \quad 2$. Disponible en: http://dx.doi.org/10.6018/analesdoc.21.2.302651. 
bibliográficas citadas en estos trabajos como indicadores de uso y se procesaron para calcular su cantidad y edad, y determinar el idioma y tipo documental.

\section{REVISIÓN DE LA LITERATURA}

En el ámbito de la bibliometría, el análisis de citas se basa en un aspecto característico de la comunicación científica: la forma en que los académicos dan cuenta de aquellos documentos que han servido de base, referencia o inspiración para su propio trabajo. Esta práctica, la de dar crédito a otros autores, da origen a una red que conecta documentos que citan con documentos que son citados (Andrés, 2009; Merton, 1979).

Esta red puede examinarse con diversos propósitos y desde diferentes ópticas: caracterizar la evolución de una disciplina a partir de sus publicaciones, identificando frentes de investigación emergentes o en camino a la obsolescencia; develar conexiones entre áreas de conocimiento, instituciones, países o regiones; presentar patrones de comunicación en la comunidad científica; o construir teorías de citación y, analizar cómo y por qué citan determinados individuos o grupos (Milojević, 2012; Smith, 1981).

En el terreno metodológico, una de las técnicas más empleadas en el análisis de citas consiste en extraer referencias bibliográficas de un conjunto de documentos y examinar sus características. Identificadas, las referencias se contabilizan y categorizan según un criterio: por ejemplo, edad (diferencia de años de publicación entre el documento que cita y el documento citado), tipo de documento referido (libro, artículo de revista, tesis, blog, etc.) o idioma. Los pioneros en utilizar esta técnica fueron Gross y Gross (1927), quienes compilaron y cuantificaron todas las referencias bibliográficas del volumen de 1926 de The Journal of the American Chemical Society, y recomendaron a la biblioteca del Pomona College (California) la compra de las 28 revistas más citadas.

Los estudios que emplean esta técnica (análisis de referencias, si cabe la precisión) pueden clasificarse en dos grandes grupos, determinados por los documentos de donde se toman las referencias bibliográficas.

a) A partir de documentos publicados en un área disciplinar (estudios sobre la literatura)

En este caso, se pretende mapear la literatura de un área del conocimiento sobre la base de lo que Ashman (2009, p. 115) denomina su "estructura bibliográfica". La premisa es que, al caracterizar las referencias de un núcleo documental disciplinar, se revelen patrones, conexiones internas y relaciones con otras áreas, y de esta forma, se delimite el saber acumulado. Las conclusiones derivadas de estos estudios pueden, entre otras cosas, identificar fuentes bibliográficas fundamentales en un área disciplinar. Vista así en retrospectiva, la bibliografía delinea futuros usos de información (Smith, 1981).

Las referencias se extraen de publicaciones consideradas prestigiosas o representativas del campo: selecciones de artículos o libros, de volúmenes de una revista o de grupos de revistas.

Estudios de este tipo se han realizado en distintas áreas: a partir de revistas en lingüística (Ezema y Asogwa, 2014), literatura (Hammarfelt, 2012), humanidades (Knievel y Kellsey, 2005), medicina (Basulto Casas y Sánchez Tarragó, 2008) (Solórzano Álvarez et al., 2006), relaciones internacionales (Griffiths, 2008; Zhang, 2007); o libros y revistas en historia (Dalton y Charnigo, 2004).

El núcleo documental disciplinar, de donde se toman las referencias, también puede determinarse considerando lo incluido en índices comerciales de citas para las ciencias naturales, ciencias sociales y humanidades (Du y Song, 2013; Glänzel y Schoepflin, 1999), (Larivière et al., 2006); o en bases de datos interdisciplinarias (Noe y Furay, 2013). Otros estudios, por su parte, se han basado en lo publicado en ciencia y tecnología por sociedades científicas (Wainer, Przibisczki de Oliveira y Anido, 2011) o por autores prestigiosos (Lascar y Mendelsohn, 2001).

b) A partir de documentos producidos (no necesariamente publicados) por poblaciones específicas de académicos (estudios de usuarios)

En este segundo grupo, el objetivo es caracterizar el uso de información de poblaciones particulares de académicos en países, universidades o centros de investigación (Ashman, 2009; Smith, 1981).

Mientras que en el primer caso se estudia la bibliografía circulante que podría ser potencialmente consultada, aquí se examina la bibliografía en trabajos propios que ha sido efectivamente consultada por ciertos usuarios (Urbano Salido, 2001). Por ello, las referencias se extraen y examinan a partir de documentos producidos en un ámbito relativamente local: 
- Artículos publicados por investigadores de un país (Creaser, Oppenheim y Summers, 2011).

- Artículos publicados por investigadores o docentes de una institución (Costas, Leeuwen y Bordons, 2012; Currie y Monroe-Gulick, 2013; Dees, 2016; Gao, 2015; Kayongo y Helm, 2009; Ke y Bronicki, 2015; Matos, 2016; Watkins y Gunapala, 2013).

- Tesis de doctorado o maestría sustentadas por estudiantes (Becker y Chiware, 2015; Brito Ocampo, Ladrón De Guevara Solís y Rosas Poblano, 2010; Mattos y Wense Dias, 2009; Rosenberg, 2015; Smyth, 2011; Tannuri de Oliveira, de Castro Silva y Moreira Garcia, 2007).

- Ensayos o trabajos académicos elaborados por estudiantes de posgrado (Bennett y Brothen, 2010; Ivins, 2013) o pregrado (Datig, 2016; Leiding, 2005; Mill, 2008).

La presente investigación, sobre académicos PUCP, se ubica en este segundo grupo.

\section{METODOLOGÍA}

Este estudio analizó listas de referencias bibliográficas. Los datos fueron recopilados y procesados de manera similar a como reportan Ke y Bronicki (2015) en su trabajo sobre hábitos de citación de académicos en psicología. Se escogió Scopus como fuente de datos por proveer el mayor conjunto de resultados de búsqueda para esta investigación (frente a alternativas como Web of Science o SciELO) y por sus facilidades para exportar referencias a hojas de cálculo.

Se identificaron todos los documentos publicados desde el año 2010 hasta el 2014, que tenían al menos un autor afiliado a la PUCP. La búsqueda configurada con estos parámetros devolvió 670 resultados. Este conjunto inicial fue sometido a depuraciones (por existencia de duplicados o errores de afiliación) y fue incrementado con nuevos registros (por alertas de Scopus recibidas hasta finales de 2015). El conjunto final de análisis, después de estas eliminaciones y adiciones, quedó delimitado a 718 documentos, los cuales fueron clasificados de acuerdo con el departamento académico de sus autores.

Como foco del presente trabajo se eligió estudiar las cuatro unidades con mayor número de docentes y mayor representación en Scopus. La Tabla I muestra la distribución del conteo.

\begin{tabular}{|c|c|}
\hline Unidad académica & $\begin{array}{l}\text { Cantidad } \\
\text { documentos }\end{array}$ \\
\hline Ciencias & 212 \\
\hline Ciencias Sociales & 22 \\
\hline Humanidades & 47 \\
\hline Ingeniería & 209 \\
\hline Otros & 228 \\
\hline Total & 718 \\
\hline
\end{tabular}

La categoría otros agrupa a los departamentos de Arquitectura, Arte y Diseño, Artes Escénicas, Administración, Gestión, Comunicaciones, Derecho, Economía, Educación, Psicología y Teología; la Escuela de Negocios CENTRUM y el Instituto de Corrosión y Protección.

En el Departamento de Ciencias se encuentran las secciones de Física, Matemática y Química; en Ciencias Sociales, están Antropología, Ciencia Política y Sociología; en Humanidades se agrupan Arqueología, Ciencias de la Información, Filosofía, Geografía y Medio Ambiente, Historia, Lingüística y Literatura, y Trabajo Social; y en Ingeniería, las ramas Civil, de Minas, Electricidad y Electrónica, Industrial, Informática, Mecánica y Telecomunicaciones.

De la población de 490 documentos pertenecientes a las cuatro unidades de estudio, se tomó una muestra estratificada. Su tamaño $(\mathrm{n}=217)$ y distribución se aprecian en la Tabla II. 


\begin{tabular}{|l|l|l|}
\hline Departamento académico & $\begin{array}{l}\text { Cantidad de } \\
\text { documentos }\end{array}$ & $\begin{array}{l}\text { Tamaño de muestra } \\
\text { documental }\end{array}$ \\
\hline Ciencias & 212 & 94 \\
\hline Ciencias Sociales & 22 & 10 \\
\hline Humanidades & 47 & 21 \\
\hline Ingeniería & 209 & 92 \\
\hline Total & $\mathbf{N}=\mathbf{4 9 0}$ & $\mathbf{n = 2 1 7}$ \\
\hline
\end{tabular}

Tabla II. Muestra documental estratificada por departamento académico.

El tamaño de la muestra fue calculado considerando los siguientes parámetros estadísticos: un porcentaje estimado de la muestra de 50\%, un margen de error (error máximo aceptable) de 5\% y un nivel de confianza de $95 \%$. Se decidió aplicar un "muestreo probabilístico estratificado" (selección aleatoria y proporcional en cada estrato) con el fin de realizar comparaciones entre subgrupos.

Del grupo de documentos categorizados por departamento $(\mathrm{N}=490)$ se excluyeron trabajos escritos en coautoría entre alguno de los cuatro departamentos bajo estudio. Este fue el caso de dos colaboraciones de Ciencias Sociales con Humanidades y dos de Ciencias con Ingeniería.

A continuación, las referencias bibliográficas presentes en la muestra documental $(n=217)$ fueron exportadas a hojas de cálculo desde Scopus y luego examinadas, separadamente por departamento académico, para determinar su cantidad, edad, idioma y tipo documental.

La edad se calculó como la diferencia, en años de publicación, entre el documento que cita y el documento que es referenciado. Se optó por mantener las referencias de gran antigüedad (fuentes primarias, obras clásicas, documentos de archivo, etc.), no extrañas en la investigación humanística, como un elemento más de posible comparación entre unidades académicas.

Como valor representativo de cada departamento, se obtuvo la media de las edades. Se determinó, además, el índice de obsolescencia, conocido también como vida media o semiperiodo de Burton y Kebler. Al estar definido como el tiempo (en años) en que se publicó la mitad de la literatura citada, su valor coincide con la mediana de la edad de las referencias. Se calculó, también, el índice de Price como el porcentaje de referencias con edades menores a cinco años.

En cada departamento, después de examinar el idioma de cada entrada bibliográfica, se obtuvo el índice de aislamiento como el porcentaje que correspondía a fuentes peruanas.

El tipo documental se asignó de acuerdo con la siguiente categorización (independientemente de si se encontraba en formato impreso o digital):

- Libro o capítulo de libro.

- Artículo de revista científica o académica.

- Ponencia o anales de congreso.

- Literatura gris y prensa general (tesis, informe, norma, memoria, manual, artículo periodístico, reporte técnico, patente, compendio estadístico, etc.).

- Otros recursos (material audiovisual, entrevista, mapa, notas de campo, sitio web, etc.).

Recuérdese que caen en la categoría de literatura gris aquellos materiales documentales producidos por entidades gubernamentales, académicas, comerciales o industriales que no se encuentran bajo el control de casas editoriales (Rucinski, 2015).

Finalmente, no debe perderse de vista que los datos, en tanto han sido recopilados de Scopus, provienen de un entorno en donde están sobrerrepresentados los aportes de las ciencias y la ingeniería en inglés, en detrimento de contribuciones de las ciencias sociales y humanidades en español (Mongeon y Paul-Hus, 2016).

\section{RESULTADOS}

Se presentan, a continuación, las cualidades del uso de información en términos de la cantidad, la edad, el idioma y el tipo documental de las fuentes bibliográficas citadas. 


\section{Cantidad}

En la muestra de 217 documentos, se encontró un total de 6715 referencias bibliográficas, cuya distribución y cantidad media, por departamento académico, se muestran en la Tabla III.

\begin{tabular}{|l|l|l|l|}
\hline $\begin{array}{l}\text { Departamento } \\
\text { académico }\end{array}$ & $\begin{array}{l}\text { Tamaño de } \\
\text { muestra } \\
\text { documental }\end{array}$ & $\begin{array}{l}\text { Cantidad de } \\
\text { referencias }\end{array}$ & $\begin{array}{l}\text { Cantidad promedio } \\
\text { de referencias por } \\
\text { documento }\end{array}$ \\
\hline Ciencias & 94 & 3207 & 34.11 \\
\hline Ciencias Sociales & 10 & 381 & 38.10 \\
\hline Humanidades & 21 & 755 & 35.95 \\
\hline Ingeniería & 92 & 2372 & 25.78 \\
\hline Total & $\mathbf{2 1 7}$ & $\mathbf{6 7 1 5}$ & $\mathbf{3 0 . 9 4}$ \\
\hline
\end{tabular}

Tabla III. Muestra documental y cantidad de referencias por departamento académico.

Puede observarse que los académicos en Ciencias Sociales citan la mayor cantidad de fuentes (38.1 referencias por documento, en promedio), seguidos de cerca por los investigadores de Humanidades (35.95) y de Ciencias (34.23).

\section{Edad, indice de obsolescencia e indice de Price}

El tratamiento estadístico de la edad arrojó los resultados que se resumen en la Tabla IV y reveló particularidades por departamento académico.

\begin{tabular}{|l|l|l|l|l|l|}
\hline $\begin{array}{l}\text { Departamento } \\
\text { académico }\end{array}$ & Mínimo & Máximo & Media & Mediana & $\begin{array}{l}\text { Desviación } \\
\text { estándar }(\boldsymbol{\sigma})\end{array}$ \\
\hline Ciencias & 0 & 90 & 9.97 & 6 & 11.68 \\
\hline Ciencias Sociales & 0 & 82 & 13.12 & 8 & 13.49 \\
\hline Humanidades & 0 & 467 & 46.20 & 13 & 102.16 \\
\hline Ingeniería & 0 & 78 & 10.44 & 7 & 10.23 \\
\hline
\end{tabular}

Tabla IV. Edad de las referencias en años por departamento académico.

En el Departamento de Ciencias, se halló que las referencias tienen, en promedio, 9.97 años y que, a pesar de la relativa amplitud del rango (90 años), los valores tienden a concentrarse en los primeros intervalos, como puede observarse en la distribución de frecuencias (Figura 1).

En lo que concierne al Departamento de Ciencias Sociales, se determinó que las referencias utilizadas tienen una antigüedad media de 13.12 años y que las edades se ubican en un rango temporal relativamente amplio (82 años), aunque con una clara agrupación en los primeros intervalos (Figura 2).

Por otro lado, el procesamiento estadístico de los datos en el Departamento de Humanidades reveló que las fuentes usadas por sus investigadores tienen una edad promedio de 46.2 años. A pesar de la amplitud temporal (467 años), se descubrió que los valores se concentran en los primeros intervalos. A diferencia de las otras unidades, donde la distribución de las edades tiene una tendencia estrictamente descendente a continuación del pico, aquí se aprecia un pequeño repunte después de la disminución abrupta en las cantidades.

En efecto, la distribución de frecuencias de las edades (Figura 3) se puede dividir en tres zonas: (1) entre 0 y 151 años (donde se localiza el 93\% de las referencias), (2) entre 152 y 286 años (sin referencias), y (3) entre 287 y 467 años $(7 \%)$. Si solo se tuvieran en cuenta las referencias cuyas edades se localizan en la primera zona - para reducir el efecto del uso de documentos primarios, la media se reduciría a 18.92 años $(\sigma=22.32$ años). Aun así, se obtendría una edad mayor a la de los otros departamentos bajo estudio.

En el Departamento de Ingeniería, se encontró que las referencias bibliográficas tienen, en promedio, 10.44 años. Cabe precisar que los valores se obtuvieron después de eliminar dos referencias de documentos del siglo XIX, que cumplían una función de carácter ilustrativo e histórico ${ }^{1}$. De haberse mantenido estos dos valores atípicos (2374 referencias en lugar de 2372), la media se hubiera situado en 10.57 años ( $\sigma=11.25$ años).

De manera similar a los casos anteriores, la distribución de frecuencias (Figura 4) muestra que los valores de las edades se concentran en los primeros intervalos. 


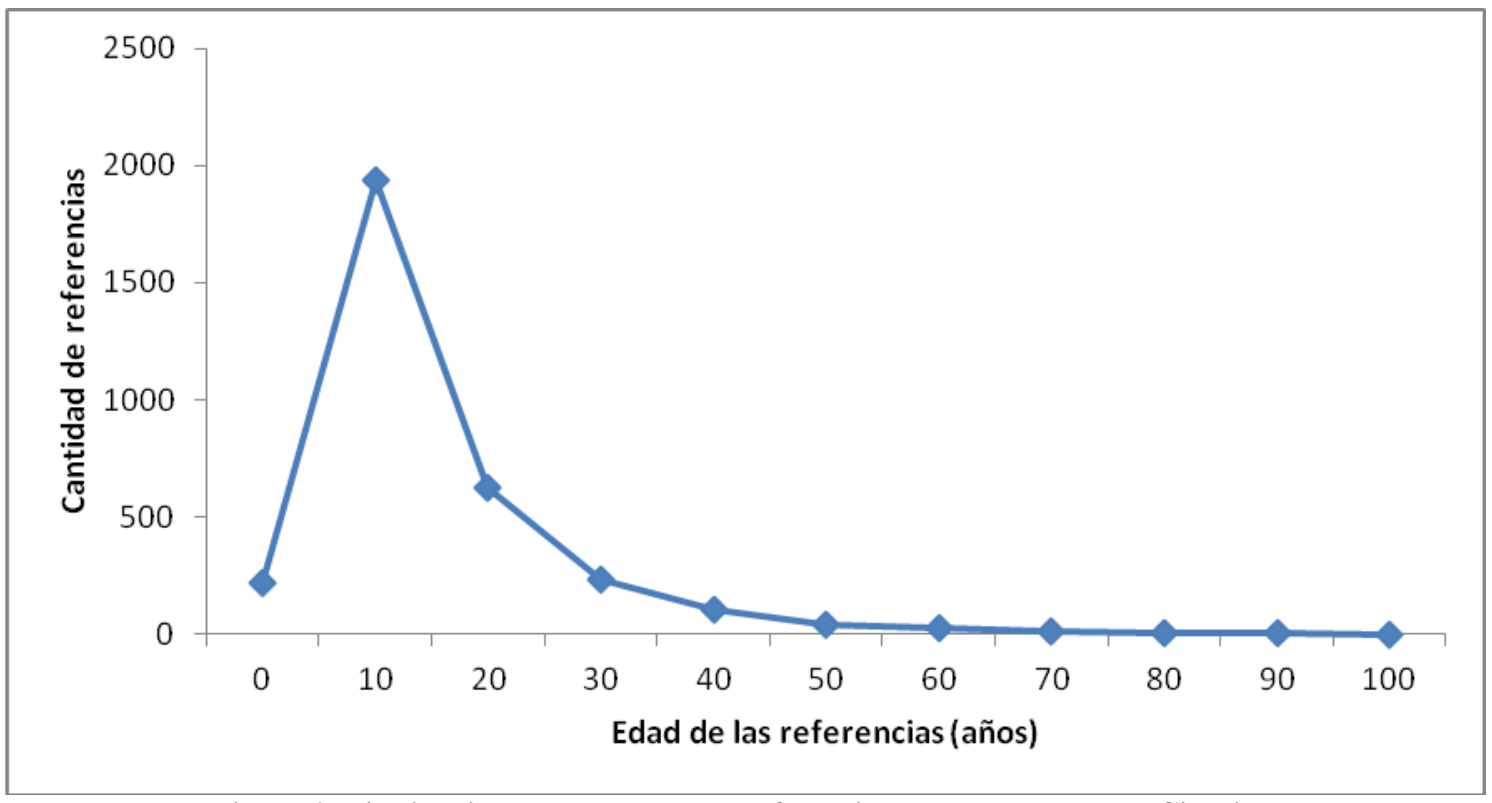

Figura 1. Distribución de la edad de las referencias - Departamento de Ciencias.

\begin{tabular}{|l|l|l|l|}
\hline $\begin{array}{l}\text { Edad } \\
\text { (años) }\end{array}$ & Cantidad & Porcentaje & $\begin{array}{l}\text { Porcentaje } \\
\text { acumulado }\end{array}$ \\
\hline 0 & 217 & $6.77 \%$ & $6.77 \%$ \\
\hline 1 & 379 & $11.82 \%$ & $18.58 \%$ \\
\hline 2 & 306 & $9.54 \%$ & $28.13 \%$ \\
\hline 3 & 231 & $7.20 \%$ & $35.33 \%$ \\
\hline 4 & 188 & $5.86 \%$ & $\mathbf{4 1 . 1 9 \%}$ \\
\hline 5 & 175 & $5.46 \%$ & $46.65 \%$ \\
\hline 6 & 149 & $4.65 \%$ & $\mathbf{5 1 . 2 9 \%}$ \\
\hline $7-20$ & 1133 & $35.33 \%$ & $86.62 \%$ \\
\hline $21-50$ & 378 & $11.79 \%$ & $98.41 \%$ \\
\hline $50-90$ & 51 & $1.59 \%$ & $100.00 \%$ \\
\hline Total & $\mathbf{3 2 0 7}$ & $\mathbf{1 0 0 . 0 0 \%}$ & \\
\hline Distribucion & la $\%$. & \\
\hline
\end{tabular}

Tabla V. Distribución de la edad de las referencias - Departamento de Ciencias. 


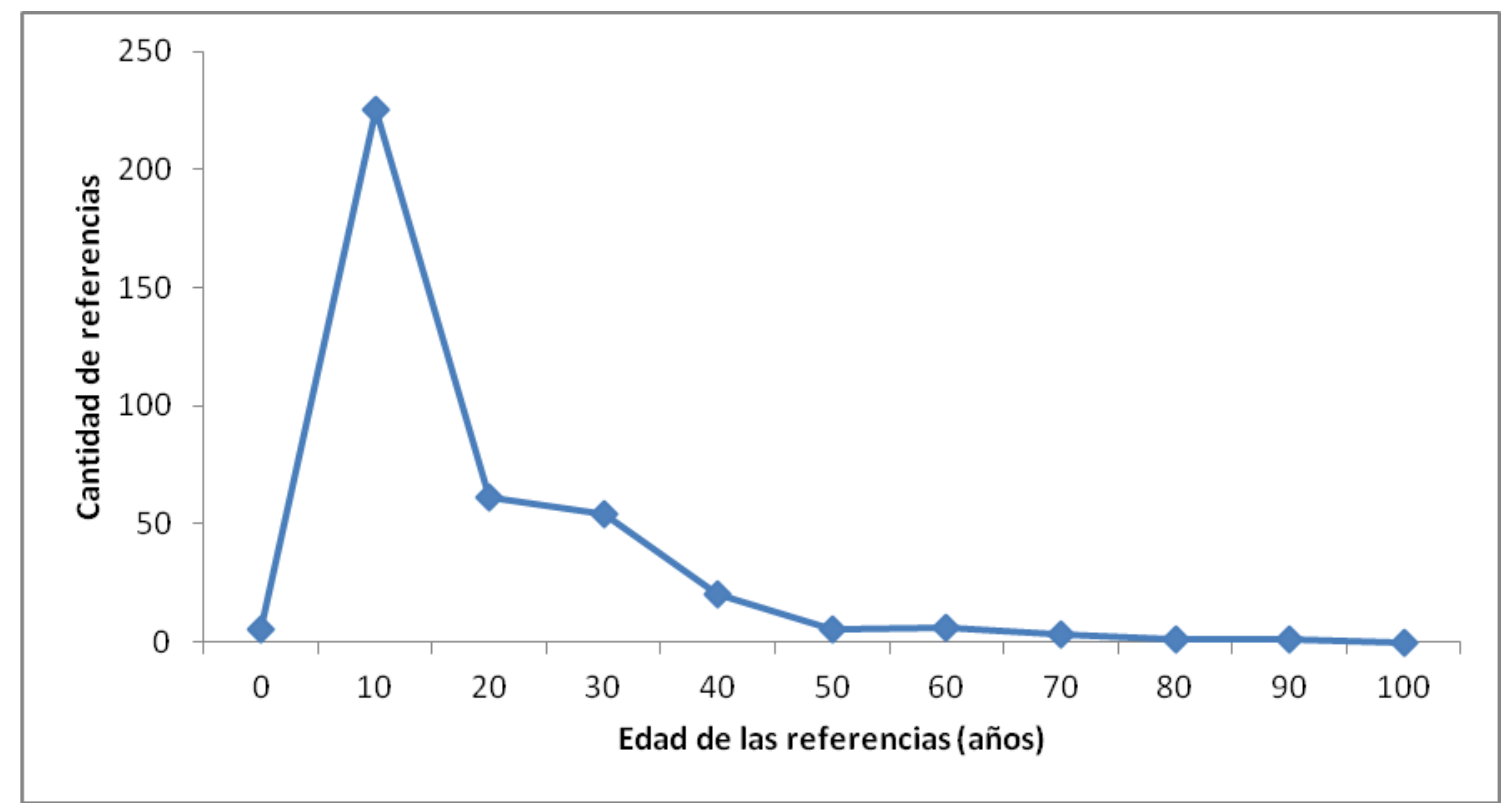

Figura 2. Distribución de la edad de las referencias - Departamento de Ciencias Sociales.

\begin{tabular}{|l|l|l|l|}
\hline $\begin{array}{l}\text { Edad } \\
\text { (años) }\end{array}$ & Cantidad & Porcentaje & $\begin{array}{l}\text { Porcentaje } \\
\text { acumulado }\end{array}$ \\
\hline 0 & 5 & $1.31 \%$ & $1.31 \%$ \\
\hline 1 & 16 & $4.20 \%$ & $5.51 \%$ \\
\hline 2 & 31 & $8.14 \%$ & $13.65 \%$ \\
\hline 3 & 33 & $8.66 \%$ & $22.31 \%$ \\
\hline 4 & 35 & $9.19 \%$ & $\mathbf{3 1 . 5 0 \%}$ \\
\hline 5 & 22 & $5.77 \%$ & $37.27 \%$ \\
\hline 6 & 18 & $4.72 \%$ & $41.99 \%$ \\
\hline 7 & 17 & $4.46 \%$ & $46.46 \%$ \\
\hline 8 & 21 & $5.51 \%$ & $\mathbf{5 1 . 9 7 \%}$ \\
\hline $9-20$ & 93 & $24.41 \%$ & $76.38 \%$ \\
\hline $21-50$ & 79 & $20.73 \%$ & $97.11 \%$ \\
\hline $51-82$ & 11 & $2.89 \%$ & $100.00 \%$ \\
\hline Total & $\mathbf{3 8 1}$ & $\mathbf{1 0 0 . 0 0 \%}$ & \\
\hline
\end{tabular}

Tabla VI. Distribución de la edad de las referencias - Departamento de Ciencias Sociales. 


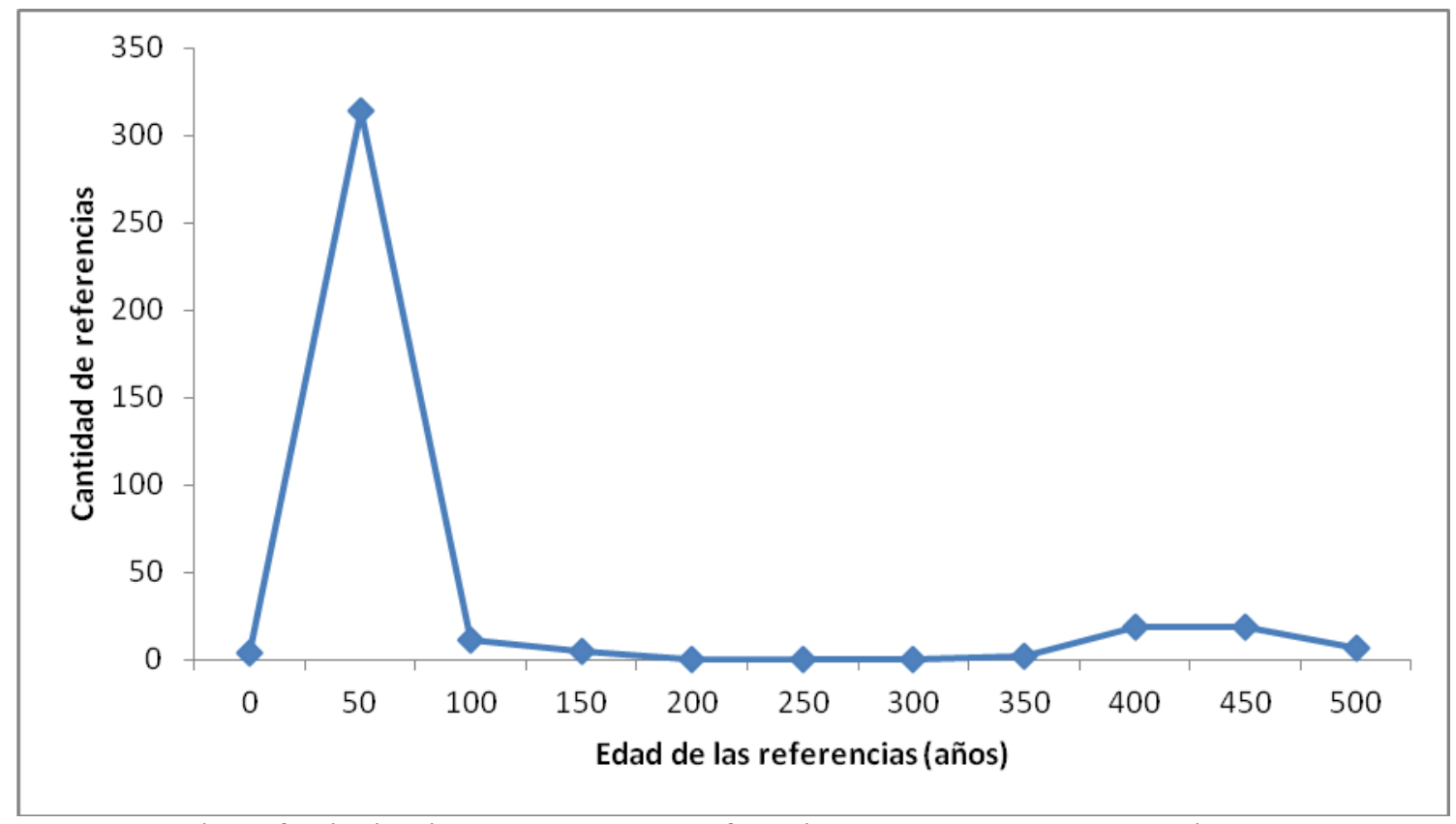

Figura 3. Distribución de la edad de las referencias - Departamento de Humanidades.

\begin{tabular}{|c|c|c|c|}
\hline $\begin{array}{l}\text { Edad } \\
\text { (años) }\end{array}$ & Cantidad & Porcentaje & $\begin{array}{l}\text { Porcentaje } \\
\text { acumulado }\end{array}$ \\
\hline 0 & 5 & $0.66 \%$ & $0.66 \%$ \\
\hline 1 & 30 & $3.97 \%$ & $4.64 \%$ \\
\hline 2 & 48 & $6.36 \%$ & $10.99 \%$ \\
\hline 3 & 33 & $4.37 \%$ & $15.36 \%$ \\
\hline 4 & 35 & $4.64 \%$ & $20.00 \%$ \\
\hline 5 & 39 & $5.17 \%$ & $25.17 \%$ \\
\hline 6 & 33 & $4.37 \%$ & $29.54 \%$ \\
\hline 7 & 30 & $3.97 \%$ & $33.51 \%$ \\
\hline 8 & 33 & $4.37 \%$ & $37.88 \%$ \\
\hline 9 & 26 & $3.44 \%$ & $41.32 \%$ \\
\hline 10 & 25 & $3.31 \%$ & $44.64 \%$ \\
\hline 11 & 22 & $2.91 \%$ & $47.55 \%$ \\
\hline 12 & 17 & $2.25 \%$ & $49.80 \%$ \\
\hline 13 & 17 & $2.25 \%$ & $52.05 \%$ \\
\hline $14-20$ & 98 & $12.98 \%$ & $65.03 \%$ \\
\hline $21-50$ & 154 & $20.40 \%$ & $85.43 \%$ \\
\hline $51-100$ & 45 & $5.96 \%$ & $91.39 \%$ \\
\hline $101-150$ & 11 & $1.46 \%$ & $92.85 \%$ \\
\hline $151-200$ & 1 & $0.13 \%$ & $92.98 \%$ \\
\hline $201-467$ & 53 & $7.02 \%$ & $100.00 \%$ \\
\hline Total & 755 & $100.00 \%$ & \\
\hline
\end{tabular}

Tabla VII. Distribución de la edad de las referencias - Departamento de Humanidades. 


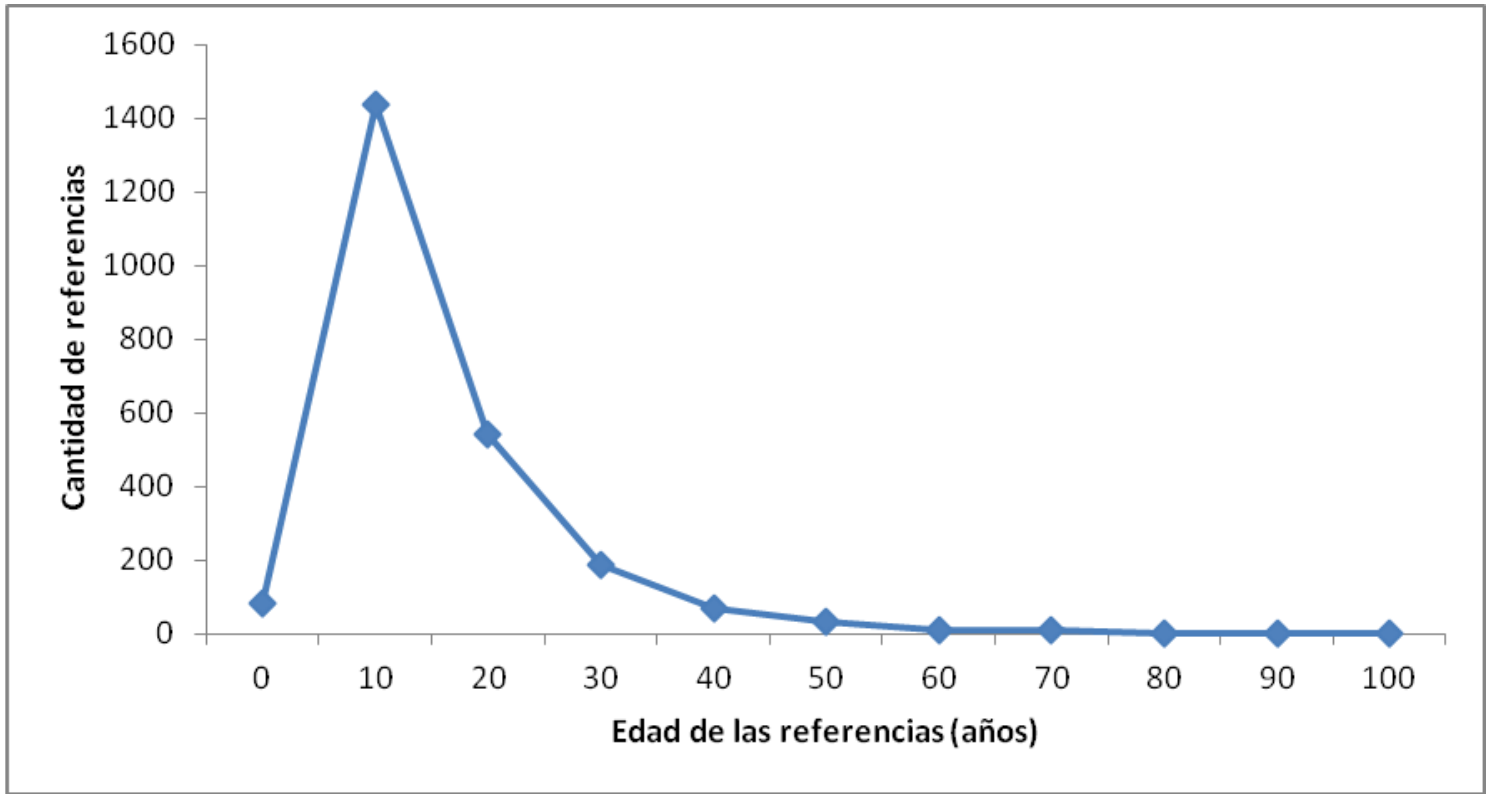

Figura 4. Distribución de la edad de las referencias - Departamento de Ingeniería.

\begin{tabular}{|l|l|l|l|}
\hline $\begin{array}{l}\text { Edad } \\
\text { (años) }\end{array}$ & Cantidad & Porcentaje & $\begin{array}{l}\text { Porcentaje } \\
\text { acumulado }\end{array}$ \\
\hline 0 & 84 & $3.54 \%$ & $3.54 \%$ \\
\hline 1 & 151 & $6.37 \%$ & $9.91 \%$ \\
\hline 2 & 195 & $8.22 \%$ & $18.13 \%$ \\
\hline 3 & 188 & $7.93 \%$ & $26.05 \%$ \\
\hline 4 & 150 & $6.32 \%$ & $\mathbf{3 2 . 3 8 \%}$ \\
\hline 5 & 168 & $7.08 \%$ & $39.46 \%$ \\
\hline 6 & 134 & $5.65 \%$ & $45.11 \%$ \\
\hline 7 & 119 & $5.02 \%$ & $\mathbf{5 0 . 1 3 \%}$ \\
\hline $8-20$ & 874 & $36.85 \%$ & $86.97 \%$ \\
\hline $21-50$ & 289 & $12.18 \%$ & $99.16 \%$ \\
\hline $51-78$ & 20 & $0.84 \%$ & $100.00 \%$ \\
\hline Total & $\mathbf{2 3 7 2}$ & $\mathbf{1 0 0 . 0 0 \%}$ & \\
\hline
\end{tabular}

Tabla VIII. Distribución de la edad de las referencias - Departamento de Ingeniería.

Los indicadores que dan cuenta de la obsolescencia de las referencias se resumen en la Tabla IX, a partir de la distribución de sus edades (Tablas V, VI, VII y VIII). Mientras que la mitad de la literatura, al momento de ser citada, tenía seis años en Ciencias, siete en Ingeniería y ocho en Ciencias Sociales, este valor se situó en trece para Humanidades. Esta dependencia en fuentes más antiguas resulta confirmada por el índice de Price: solo un 20\% de sus referencias tenía menos de cinco años, frente al 31.5\% de Ciencias Sociales, al 32.38\% de Ingeniería y al 41.19\% de Ciencias.

\begin{tabular}{|l|l|l|}
\hline Departamento académico & $\begin{array}{l}\text { Índice de obsolescencia } \\
\text { (años) }\end{array}$ & Índice de Price \\
\hline Ciencias & 6 & $41.19 \%$ \\
\hline Ciencias Sociales & 8 & $31.50 \%$ \\
\hline Humanidades & 13 & $20.00 \%$ \\
\hline Ingeniería & 7 & $32.38 \%$ \\
\hline
\end{tabular}

Tabla IX. Indicadores de obsolescencia de la literatura por departamento académico. 


\section{Idioma e índice de aislamiento}

La Tabla X muestra los idiomas de las referencias. Es considerable, en el conteo general, el predominio del inglés $(85.35 \%)$ y esta preeminencia es aún mayor para el Departamento de Ciencias $(97.75 \%)$ y para Ingeniería $(90.81 \%)$. Solo en Ciencias Sociales, el uso del español es mayoritario (84.51\%).

En la categoría varios, se clasificó la referencia al Ritvale sev Manvale Pervanvm, un documento religioso de comienzos del siglo XVII, que contiene textos en latín, español, quechua, aimara, puquina, guaraní y mochica, citado por un estudio lingüístico.

Las referencias también fueron examinadas para determinar cuáles correspondían a fuentes provenientes del Perú con el fin de calcular el índice de aislamiento. Los valores encontrados se muestran en la Tabla XI. Se observa que, mientras que Ingeniería y, en mayor medida Ciencias, prácticamente, solo consumen literatura extranjera, los académicos en Ciencias Sociales basan sus investigaciones en fuentes principalmente locales (61.9\%). 


\begin{tabular}{|c|c|c|c|c|c|c|c|c|c|c|}
\hline \multirow[t]{2}{*}{ IDIOMA } & \multicolumn{2}{|c|}{ CIENCIAS } & \multicolumn{2}{|c|}{ CIENCIAS SOCIALES } & \multicolumn{2}{|c|}{ HUMANIDADES } & \multicolumn{2}{|c|}{ INGENIERÍA } & \multicolumn{2}{|c|}{ TOTAL } \\
\hline & Cantidad & Porcentaje & Cantidad & Porcentaje & Cantidad & Porcentaje & Cantidad & Porcentaje & Cantidad & Porcentaje \\
\hline INGLÉS & 3135 & $97.75 \%$ & 52 & $13.65 \%$ & 390 & $51.66 \%$ & 2154 & $90.81 \%$ & 5731 & $85.35 \%$ \\
\hline ESPAÑOL & 32 & $1.00 \%$ & 322 & $84.51 \%$ & 289 & $38.28 \%$ & 204 & $8.60 \%$ & 847 & $12.61 \%$ \\
\hline ALEMÁN & 9 & $0.28 \%$ & & & 58 & $7.68 \%$ & 1 & $0.04 \%$ & 68 & $1.01 \%$ \\
\hline FRANCÉS & 21 & $0.65 \%$ & 4 & $1.05 \%$ & 15 & $1.99 \%$ & 2 & $0.08 \%$ & 42 & $0.63 \%$ \\
\hline PORTUGUÉS & 8 & $0.25 \%$ & 3 & $0.79 \%$ & 1 & $0.13 \%$ & 8 & $0.34 \%$ & 20 & $0.30 \%$ \\
\hline ITALIANO & 1 & $0.03 \%$ & & & 1 & $0.13 \%$ & 1 & $0.04 \%$ & 3 & $0.04 \%$ \\
\hline NORUEGO & & & & & & & 2 & $0.08 \%$ & 2 & $0.03 \%$ \\
\hline RUSO & 1 & $0.03 \%$ & & & & & & & 1 & $0.01 \%$ \\
\hline VARIOS & & & & & 1 & $0.13 \%$ & & & 1 & $0.01 \%$ \\
\hline TOTAL & 3207 & $100.00 \%$ & 381 & $100.00 \%$ & 755 & $100.00 \%$ & 2372 & $100.00 \%$ & 6715 & $100.00 \%$ \\
\hline
\end{tabular}




\begin{tabular}{|l|l|l|l|}
\hline $\begin{array}{l}\text { Departamento } \\
\text { académico }\end{array}$ & $\begin{array}{l}\text { Cantidad de } \\
\text { referencias } \\
\text { peruanas }\end{array}$ & $\begin{array}{l}\text { Cantidad total } \\
\text { de referencias }\end{array}$ & $\begin{array}{l}\text { Índice de } \\
\text { aislamiento }\end{array}$ \\
\hline Ciencias & 10 & 3207 & $0.3 \%$ \\
\hline Ciencias Sociales & 236 & 381 & $61.9 \%$ \\
\hline Humanidades & 135 & 755 & $17.9 \%$ \\
\hline Ingeniería & 101 & 2372 & $4.3 \%$ \\
\hline Total & $\mathbf{4 8 3}$ & $\mathbf{6 7 1 5}$ & $\mathbf{7 . 2 \%}$ \\
\hline
\end{tabular}

Tabla XI. Índice de aislamiento por departamento académico.

Tipo documental

El análisis documental de las referencias en las respectivas muestras halló, como en el acápite anterior, rasgos distintivos en cada unidad (Tabla XII).

En el Departamento de Ciencias, se encontró que el 79\% de las referencias está constituido por artículos de revista. El segundo tipo de documento más frecuentemente referenciado — aunque de forma mucho más modesta con un $8.3 \%$ - está representado por las comunicaciones en congresos.

Por su parte, en el Departamento de Ciencias Sociales, se halló que los libros o capítulos de libro constituyen el tipo de fuente citado con mayor frecuencia. Así sucede en el 47.2\% de los casos. A continuación, se ubicó la literatura gris y prensa general con un 36\%, con documentos en forma de tesis, artículos periodísticos de diarios o revistas no académicas, informes de organismos no gubernamentales, publicaciones oficiales, compendios estadísticos y normas legales. De otro lado, se descubrió una relativamente baja proporción de referencias a artículos de revistas (11.8\%).

En el Departamento de Humanidades, se encontró que los investigadores hacen referencia, en casi la mitad de los casos (48.9\%), a libros o capítulos de libro y en segundo lugar, a artículos de revista (37.9\%).

Finalmente, en el Departamento de Ingeniería, se halló que el artículo de revista es el vehículo preferido de comunicación con un $57.2 \%$ de las referencias, aunque no de manera tan prominente como en Ciencias. Asimismo, se encontró que las comunicaciones a través de congresos representan la segunda categoría documental más significativa, en un porcentaje que supera largamente a las otras unidades bajo estudio $(19.6 \%$ versus $8.3 \%$ en Ciencias, $2.5 \%$ en Humanidades y $2.4 \%$ en Ciencias Sociales). 


\begin{tabular}{|c|c|c|c|c|c|c|c|c|c|c|}
\hline \multirow[t]{2}{*}{ Tipo documental } & \multicolumn{2}{|l|}{ Ciencias } & \multicolumn{2}{|c|}{ Ciencias Sociales } & \multicolumn{2}{|c|}{ Humanidades } & \multicolumn{2}{|l|}{ Ingeniería } & \multicolumn{2}{|l|}{ Total } \\
\hline & Cantidad & Porcentaje & Cantidad & Porcentaje & Cantidad & Porcentaje & Cantidad & Porcentaje & Cantidad & Porcentaje \\
\hline Artículo de revista académica & 2533 & $79.0 \%$ & 45 & $11.8 \%$ & 286 & $37.9 \%$ & 1356 & $57.2 \%$ & 4220 & $62.8 \%$ \\
\hline Libro o capítulo de libro & 141 & $4.4 \%$ & 180 & $47.2 \%$ & 369 & $48.9 \%$ & 190 & $8.0 \%$ & 880 & $13.1 \%$ \\
\hline $\begin{array}{llll}\begin{array}{l}\text { Literatura } \\
\text { general }\end{array} & \text { gris } & \text { y } & \text { prensa } \\
\end{array}$ & 233 & $7.3 \%$ & 137 & $36.0 \%$ & 73 & $9.7 \%$ & 261 & $11.0 \%$ & 704 & $10.5 \%$ \\
\hline $\begin{array}{l}\begin{array}{l}\text { Anales } \\
\text { congreso }\end{array}\end{array}$ & 265 & $8.3 \%$ & 9 & $2.4 \%$ & 19 & $2.5 \%$ & 464 & $19.6 \%$ & 757 & $11.3 \%$ \\
\hline Otros recursos & 35 & $1.1 \%$ & 10 & $2.6 \%$ & 8 & $1.1 \%$ & 101 & $4.3 \%$ & 154 & $2.3 \%$ \\
\hline Total & 3207 & $100.0 \%$ & 381 & $100.0 \%$ & 755 & $100.0 \%$ & 2372 & $100.0 \%$ & 6715 & $100.0 \%$ \\
\hline
\end{tabular}

Tabla XII. Distribución de las referencias por tipo documental y departamento académico. 


\section{DISCUSIÓN}

La literatura sobre la conducta informativa reporta que los hábitos y preferencias de los académicos están condicionados por factores disciplinares (Bouazza, 1989; Hernández Salazar, 2001; Sanz Casado, 1994).

Las cifras compiladas en las tablas XIII, XIV y XV a manera de referencia muestran, con algunas excepciones, cierto nivel de correspondencia entre los resultados de este estudio y los de investigaciones similares.

\begin{tabular}{|c|c|c|}
\hline Área disciplinar & $\begin{array}{l}\text { Cantidad promedio de } \\
\text { referencias } \mathrm{PUCP}\end{array}$ & $\begin{array}{l}\text { Cantidad promedio de referencias en } \\
\text { otros estudios } \\
\text { (Glänzel y Schoepflin, 1999) }\end{array}$ \\
\hline Ciencias & 34.11 & $\begin{array}{l}23.6 \text { en física del estado sólido } \\
20.9 \text { en química analítica } \\
16.2 \text { en matemática }\end{array}$ \\
\hline Ciencias Sociales & 38.10 & 32.7 en sociología \\
\hline Humanidades & 35.95 & 48.7 en historia y filosofía de la ciencia \\
\hline Ingeniería & 25.78 & 15.0 en ingeniería \\
\hline
\end{tabular}

Tabla XIII. Cantidad de referencias en otros estudios.

\begin{tabular}{|l|l|l|}
\hline Área disciplinar & $\begin{array}{l}\text { Edad promedio de las } \\
\text { referencias PUCP (años) }\end{array}$ & $\begin{array}{l}\text { Edad promedio de referencias en otros } \\
\text { estudios } \\
\text { (Glänzel y Schoepflin, 1999) }\end{array}$ \\
\hline Ciencias & 9.97 & $\begin{array}{l}10.1 \text { en física del estado sólido } \\
9.4 \text { en química analítica } \\
11.3 \text { en matemática }\end{array}$ \\
\hline Ciencias Sociales & 13.12 & 12.5 en sociología \\
\hline Humanidades & 46.20 & $\begin{array}{l}38.8 \text { años en historia y filosofía de la } \\
\text { ciencia y las ciencias sociales }\end{array}$ \\
\hline Ingeniería & 10.44 & \begin{tabular}{l}
8.6 en ingeniería electrónica \\
\hline
\end{tabular} \\
\hline
\end{tabular}

Considerando los hallazgos de la sección anterior, puede afirmarse que cada departamento PUCP presenta características particulares en términos de la cantidad, edad, idioma y tipo documental de las fuentes citadas por sus académicos. 


\begin{tabular}{|c|c|c|c|}
\hline Tipo documental & Área disciplinar & $\begin{array}{l}\text { Porcentaje de } \\
\text { referencias PUCP }\end{array}$ & Porcentaje de referencias en otros estudios \\
\hline \multirow{4}{*}{$\begin{array}{l}\text { Artículo de } \\
\text { revista académica }\end{array}$} & Ciencias & $79.0 \%$ & $\begin{array}{l}86 \% \text { en ciencias (Tucker, } 2013 \text { ) } \\
82.3 \% \text { en física, } 84.6 \% \text { en química, } 70.8 \% \text { en matemática (Larivière et al., 2006) } \\
85.2 \% \text { en física del estado sólido, } 83.8 \% \text { en química analítica, } 64.4 \% \text { en matemática (Glänzel y Schoepflin, } \\
1999 \text { ) }\end{array}$ \\
\hline & Ciencias Sociales & $11.8 \%$ & $\begin{array}{l}40.4 \% \text { en sociología (Glänzel y Schoepflin, } 1999) \\
51.7 \% \text { en economía y gestión (Larivière } \text { et al., 2006) } \\
30.4 \% \text { en ciencias sociales (Romanos de Tiratel, 2000) }\end{array}$ \\
\hline & Humanidades & $37.9 \%$ & $\begin{array}{l}34.1 \% \text { en historia, } 23 \% \text { en literatura (Larivière et al., 2006) } \\
35.9 \% \text { en lingüística (Ezema y Asogwa, 2014) } \\
23.3 \% \text { en historia, } 37.6 \% \text { en lingüística, } 16.6 \% \text { en literatura, } 48.2 \% \text { en filosofía (Knievel y Kellsey, 2005) } \\
22.2 \% \text { en humanidades (Romanos de Tiratel, 2000) }\end{array}$ \\
\hline & Ingeniería & $57.2 \%$ & $\begin{array}{l}64.8 \% \text { en ingeniería (Larivière et al., 2006) } \\
66 \% \text { en ingeniería (Tucker, 2013) } \\
62.2 \% \text { en ingeniería electrónica (Glänzel y Schoepflin, 1999) } \\
85.9 \% \text { en ingeniería aeroespacial (Du y Song, 2013) }\end{array}$ \\
\hline \multirow{2}{*}{$\begin{array}{l}\text { Libro o capítulo } \\
\text { de libro }\end{array}$} & Ciencias Sociales & $47.2 \%$ & $\begin{array}{l}83 \% \text { en ciencia política y } 72.31 \% \text { en sociología (Huang y Chang, 2008) } \\
81 \% \text { en ciencia política (Nederhof, van Leeuwen y van Raan, 2010) } \\
47.1 \% \text { en ciencias sociales (Romanos de Tiratel, 2000) }\end{array}$ \\
\hline & Humanidades & $48.9 \%$ & $\begin{array}{l}51.4 \% \text { en filosofía, } 83 \% \text { en literatura, } 60.8 \% \text { en lingüística, } 76.4 \% \text { en historia (Knievel y Kellsey, 2005) } \\
53.3 \% \text { en lingüística (Ezema y Asogwa, 2014) } \\
62 \% \text { en humanidades (Romanos de Tiratel, 2000) }\end{array}$ \\
\hline $\begin{array}{lr}\text { Anales } & \text { o } \\
\text { ponencia } & \text { en } \\
\text { congreso } & \end{array}$ & Ingeniería & $19.6 \%$ & $\begin{array}{l}13.1 \% \text { en ingeniería eléctrica y electrónica, } 11.5 \% \text { en ingeniería civil, } 8.5 \% \text { en ingeniería industrial, } 8 \% \text { en } \\
\text { ingeniería mecánica (Lisée, Larivière y Archambault, 2008) } \\
40 \% \text { en ciencias de la computación (Wainer, Przibisczki de Oliveira y Anido, 2011) }\end{array}$ \\
\hline
\end{tabular}

Tabla XV. Tipo documental de las referencias en otros estudios. 
En el Departamento de Ciencias, los artículos de revista son el tipo de fuente más consultado en un porcentaje prominente $(79 \%)$, de forma similar a lo reportado en otros estudios (Tabla XV). La edad de las referencias está representada por una media de 9.97 años y una mediana de 6. En el Departamento de Ingeniería, se usan fuentes de una antigüedad ligeramente mayor (una media de 10.44 años y una mediana de 7) que provienen, también en su mayoría, de revistas (57.2\%). Esta combinación de factores perfila a los investigadores de ambas unidades como consumidores de literatura reciente con cortos periodos de publicación. Sus índices de aislamiento $(0.3 \%$ y $4.3 \%$, respectivamente) revelan, además, una dependencia casi total de fuentes en inglés (97.75\% y $90.81 \%)$, lo cual es consistente con bajísimos niveles de producción local en ciencia y tecnología.

Solo por propósitos de comparación en lo que a edad de la literatura concierne, cabe señalar que las revistas más consultadas son Physical Review Letters en Ciencias (274 referencias) y Journal of the Acoustical Society of America (68 referencias) en Ingeniería. Según Clarivate Analytics, la mediana de las edades de los artículos citados por estas publicaciones (citing half-life) fue de 6.4 en 2010 y 7.2 en 2014 en el primer caso, y de más de 10 años en el periodo 2010-2014, en el segundo.

Hasta aquí, podría afirmarse que se trata de grupos relativamente semejantes, pero las proporciones en las otras categorías documentales en las obras citadas (Figura 5) marcan ciertas distancias y sugieren estilos de comunicación diferentes. El descubrimiento más interesante en el Departamento de Ingeniería es el rol que desempeñan los congresos, con un $20 \%$ de referencias a ponencias o anales. Este porcentaje es relativamente superior a los valores documentados por otros análisis de citas en ramas ingenieriles (Tabla XV), a excepción de las ciencias de computación (Vrettas y Sanderson, 2015), donde los valores llegan a 40\% (Wainer, Przibisczki de Oliveira y Anido, 2011).

Se puede especular que en los congresos se toma conocimiento del estado del arte, de una forma eficiente, a través de presentaciones concisas y especializadas. Ello es consistente con la idea de que los ingenieros prefieren fuentes que los ayuden a resolver un problema de forma inmediata, a diferencia de los científicos cuyas búsquedas incluyen también información de contexto (Pinelli, 2001). Por otro lado, Pinelli (1991, p. 13), citando investigaciones previas, afirmaba que los ingenieros tienen predisposición "a resolver problemas solos o con la ayuda de otros, en lugar de buscar las respuestas en la literatura". Ello explicaría el menor uso de referencias bibliográficas en los trabajos de este grupo (Tabla III).

En este entorno, donde las nuevas investigaciones se basan en datos y hechos de reciente descubrimiento y se nutren de teorías en desarrollo, resulta comprensible la reducida dependencia en libros o capítulos de libros (4.4\% en Ciencias y $8 \%$ en Ingeniería), en claro contraste con los otros dos departamentos académicos (Figura 5).

En efecto, en el Departamento de Ciencias Sociales, los libros son la fuente que se usa casi la mitad de las veces (47.2\%). Este valor coincide con investigaciones sobre científicos sociales en el ámbito latinoamericano (47.1\%), pero no en otros casos, donde los valores están en el rango del 80\% (Tabla XV). Los libros vienen seguidos no muy lejos por los documentos grises $(36 \%)$. Esto podría reflejar la necesidad de una literatura un tanto más consolidada en el campo, y, además, complementada por información que no tiene un carácter estrictamente académico o que no se difunde necesariamente por canales científicos. El uso de la literatura gris es, pues, un rasgo distintivo de este grupo. Ello tiene sentido si se tiene en cuenta que el estudio de comportamientos e interacciones humanas en las ciencias sociales tiene una orientación mayormente local (Archambault et al., 2006), y esta delimitación geográfica y cronológica precisa de información de carácter coyuntural (estadísticas, noticias, reportajes, etc.) y relativamente reciente. Este amplio abanico de fuentes, donde sobresalen los recuentos de origen peruano (61.9\%) y de primera mano, estaría - al menos, en parte — contribuyendo a conformar bibliografías más extensas que en las otras unidades (Tabla III).

El porcentaje de artículos de revistas $(11.8 \%)$ representa, sin embargo, una marcada diferencia respecto a los valores reportados en la literatura, que los sitúa alrededor del $40 \%$ (Tabla XV).

Finalmente, como sus pares en Ciencias Sociales, los investigadores del Departamento de Humanidades citan libros prácticamente la mitad de las veces (48.9\%), pero consultan más artículos de revista (37.9\% versus $11.8 \%)$. Esta dependencia de publicaciones tanto monográficas como periódicas se encuentra bien documentada (Stone, 1982) y reflejaría la necesidad de una cobertura retrospectiva relativamente amplia.

Con referencias que tienen una edad promedio de 46.2 años y una dispersión temporal de más de cuatro siglos, se concluye que la literatura consultada en este departamento es la menos susceptible de obsolescencia en comparación con la de otras unidades. Así lo muestra un conjunto de fuentes con un índice de obsolescencia de trece años, 
claramente distante de los valores de entre seis y ocho años de los otros departamentos. Además de discutir temas universales que no pierden vigencia o interpretar acontecimientos actuales a la luz de teorías clásicas, el trabajo humanístico consiste en estudiar obras o artefactos artísticos, históricos o culturales de cualquier época. No debería extrañar, pues, el hallazgo de referencias lejanas en el tiempo. De hecho, en la muestra documental analizada, 51 citas correspondían a crónicas, memorias, catecismos, sermones y recopilaciones lexicográficas (de quechua y aimara) de los siglos XVI y XVII, presentes en trabajos de historiadores y lingüistas.

Un rasgo interesante es que, a diferencia de las otras tres unidades, no existe un marcado desbalance en el idioma de las fuentes. Un $38.28 \%$ de referencias en español, actuando como contrapeso al inglés (51.6\%), estaría revelando un consumo moderado de contenidos regionales y locales.

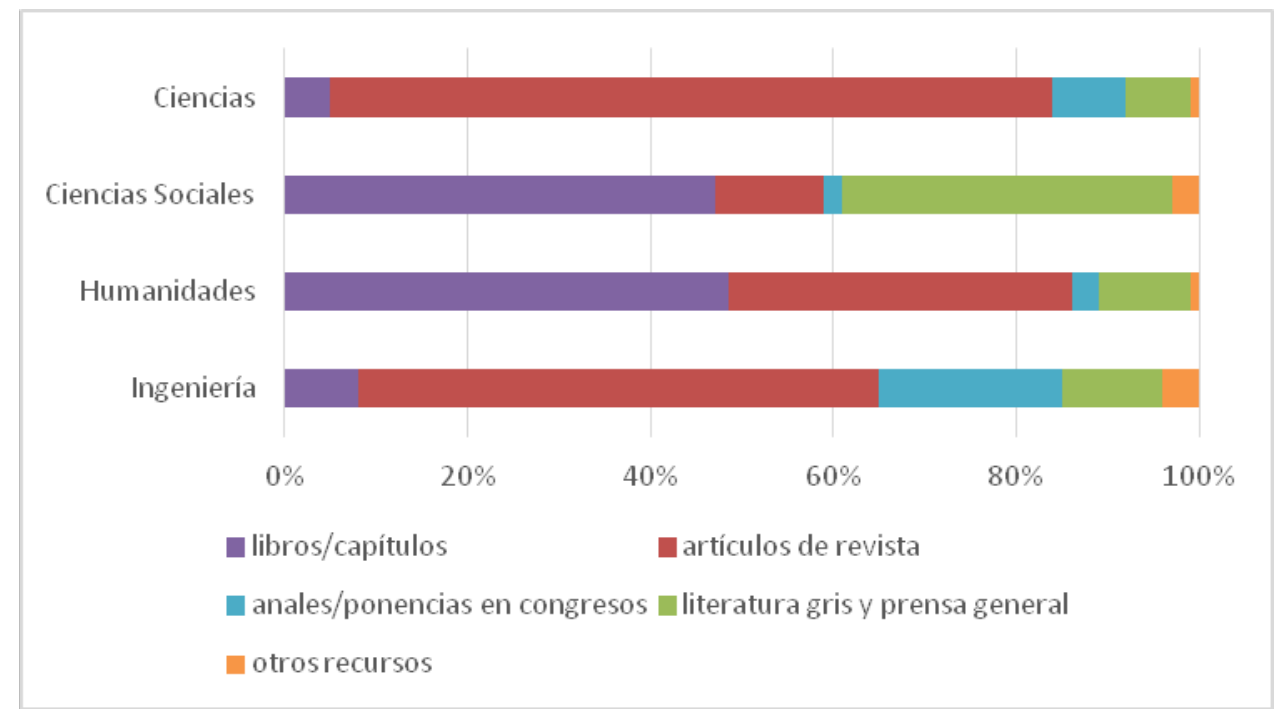

Figura 5. Tipo documental de las referencias por departamento académico.

\section{CONCLUSIONES}

Se concluye que cada departamento académico PUCP exhibe diferencias en el uso de información, específicamente en relación con las características y naturaleza de las fuentes citadas por los investigadores. Ello abonaría la presunción de que los factores disciplinares son determinantes — tal vez, los más influyentes— en el uso de información entre académicos.

El panorama encontrado lleva a pensar que las preferencias por ciertos canales de difusión del conocimiento, así como su alcance geográfico y cronológico, están determinadas por patrones de comunicación propios en cada grupo, por la naturaleza del objeto de estudio y, en esencia, en un nivel más fundamental, por la disciplina misma. En resumen, son destacables la preeminencia de literatura relativamente reciente en las ciencias exactas y naturales, la importancia de los congresos para las ingenierías, el uso intensivo de referencias locales de primera mano en las ciencias sociales y la amplitud temporal de las fuentes en las humanidades.

De cara a futuros estudios, la caracterización del uso de información en cada departamento académico PUCP podría completarse con el análisis de otras variables como el grado de autocitación de los autores. Por otro lado, si se recuerda que en los departamentos hay subdivisiones, un estudio que considere áreas de especialización podría identificar necesidades específicas en cada grupo y determinar en qué medida estas son cubiertas por los recursos que brinda la biblioteca. Adicionalmente, se podría indagar cualitativamente cómo los investigadores buscan y localizan información, qué barreras encuentran, qué recursos consultan, etc. El uso de SciELO como herramienta complementaria de datos bibliográficos (más allá del alcance planeado para esta investigación), hubiera contribuido a trazar un panorama más completo del fenómeno en áreas subrepresentadas por Scopus o Web of Science.

Se espera que la evidencia aquí presentada brinde elementos de juicio para tomar decisiones sobre colecciones, recursos y servicios de información, $\mathrm{y}$, en otro plano, sobre políticas e incentivos de investigación institucionales que consideren las diferencias entre áreas disciplinares. 


\section{NOTAS}

${ }^{1}$ Illustrations of the Huttonian Theory of the Earth (1802) de Playfair y Cours d'Economie Politique (1896) de Pareto.

\section{Agradecemientos}

La autora desea agradecer a la profesora Aurora de la Vega (PUCP) sus valiosos comentarios sobre versiones tempranas de este manuscrito.

\section{BIBLIOGRAFÍA}

ANDRÉS, A. Measuring academic research: How to undertake a bibliometric study. Oxford: Chandos Publishing, 2009.

ARCHAMBAULT, É. et al. Benchmarking scientific output in the social sciences and humanities: The limits of existing databases. Scientometrics, 2006, vol. 68, n 3, p. 329-342.

ASHMAN, A.B. An examination of the research objectives of recent citation analysis studies. Collection Management, 2009, vol. 34, $\mathrm{n}^{\circ}$ 2, p. 112-128.

BASULTO CASAS, A. y SÁNCHEZ TARRAGÓ, N. Utilización de las publicaciones científicas electrónicas por los investigadores cubanos en el área de la Higiene y la Epidemiología. ACIMED, 2008, vol. 18, no 1, p. 1-14 [en línea]. Disponible en: <http://scielo.sld.cu/scielo.php?script=sci_arttext\&pid=S1024-94352008000700004> [Consulta: 8 de febrero de 2018].

BECKER, D.A. y CHIWARE, E.R.T. Citation analysis of masters' theses and doctoral dissertations: Balancing library collections with students' research information needs. The Journal of Academic Librarianship, 2015, vol. 41, $\mathrm{n}^{\circ}$ 5, p. 613-620.

BENNETT, E. y BROTHEN, E. Citation analyses as a prioritization tool for instruction program development. Journal of Library Administration, 2010, vol. 50, n 5-6, p. 425-442.

BOUAZZA, A. Information user studies. Encyclopedia of Library and Information Science. New York: Mercel Deker, 1989, p. 144-164.

BRITO OCAMPO, L.B.; LADRÓN DE GUEVARA SOLÍS, M. del P. y ROSAS POBLANO, S.G. Impacto de la biblioteca universitaria en los estudios de posgrado: análisis de citas de las tesis en tres programas de maestría y doctorado en la Universidad Autónoma de México (UNAM). Ibersid, 2010, vol. 4, p. 195-199.

COSTAS, R.; LEEUWEN, T.N. y BORDONS, M. Referencing patterns of individual researchers: Do top scientists rely on more extensive information sources? Journal of the American Society for Information Science and Technology, 2012, vol. 63, $\mathrm{n}^{\circ}$ 12, p. 2433-2450.

CREASER, C.; OPPENHEIM, C. y SUMMERS, M. What do UK academics cite? An analysis of references cited in UK scholarly outputs. Scientometrics, 2011, vol. 86, no 3, p. 613-627.

CURRIE, L. y MONROE-GULICK, A. What do our faculty use? An interdisciplinary citation analysis study. Journal of Academic Librarianship, 2013, vol. 39, nº 6, p. 471-480.

DALTON, M.S. y CHARNIGO, L. Historians and their information sources. College \& Research Libraries, 2004, vol. $65, n^{\circ} 5$, p. $400-425$.

DATIG, I. Citation behavior of advanced undergraduate students in the social sciences: A mixed-method approach. Behavioral \& Social Sciences Librarian, 2016, vol. 35, $\mathrm{n}^{\circ}$ 2, p. 64-80.

DEES, A.S. A business citation analysis: A local follow-up study. Journal of Business \& Finance Librarianship, 2016, vol. 21, no 1 , p. 49-59.

DU, W. y SONG, S. Information needs analysis of the aerospace discipline. Aslib Proceedings, 2013, vol. 65, nº 4, p. 376-387.

EZEMA, I.J. y ASOGWA, B.E. Citation analysis and authorship patterns of two linguistics journals. Libraries and the Academy, 2014, vol. 14, no 1, p. 67-85.

GAO, W. Information use in communication research: A citation analysis of faculty publication at the University of Houston. Behavioral \& Social Sciences Librarian, 2015, vol. 34, no 3, p. 116-128.

GLÄNZEL, W. y SCHOEPFLIN, U. A bibliometric study of reference literature in the sciences and social sciences. Information Processing \& Management, 1999, vol. 35, $\mathrm{n}^{\circ}$ 1, p. 31-44.

GRIFFITHS, D.N. The citation of United Nations information resources in international relations literature. Behavioral \& Social Sciences Librarian, 2008, vol. 26, $\mathrm{n}^{\circ}$ 4, p. 1-19.

GROSS, P.L.K. y GROSS, E.M. College libraries and chemical education. Science, 1927, vol. 66, no 1713, p. 385389.

HAMMARFELT, B. Harvesting footnotes in a rural field: Citation patterns in Swedish literary studies. Journal of Documentation, 2012, vol. 68, $\mathrm{n}^{\circ}$ 4, p. 536-558. 
HERNÁNDEZ SALAZAR, P. La producción del conocimiento científico como base para determinar perfiles de usuarios. Investigación Bibliotecológica, 2001, vol. 15, nº 30, p. 29-64.

HUANG, M. y CHANG, Y. Characteristics of research output in social sciences and humanities: From a research evaluation perspective. Journal of the American Society for Information Science and Technology, 2008, vol. 59, $\mathrm{n}^{\circ}$ 11, p. 1819-1828.

IVINS, T. A case study of periodical use by Library and Information Science students. Journal of Education for Library and Information Science, 2013, vol. 54, n 2, p. 124-134.

KAYONGO, J. y HELM, C. Citation patterns of the faculty of the Anthropology Department at the University of Notre Dame. Behavioral \& Social Sciences Librarian, 2009, vol. 28, nº 3, p. 87-99.

KE, I. y BRONICKI, J. Using Scopus to study researchers' citing behavior for local collection decisions: A focus on psychology. Journal of Library Administration, 2015, vol. 55, nº 3, p. 165-178.

KNIEVEL, J. y KELLSEY, C. Citation analysis for collection development: A comparative study of eight humanities fields. The Library Quarterly: Information, Community, Policy, 2005, vol. 75, n 2, p. 142-168.

LARIVIERE, V.; ARCHAMBAULT, É.; GINGRAS, Y. y VIGNOLA-GAGNÉ, É. The place of serials in referencing practices: Comparing natural sciences and engineering. Journal of the American Society for Information Science and Technology, 2006, vol. 57, $\mathrm{n}^{\circ} 8, \mathrm{p} .997-1004$

LASCAR, C. y MENDELSOHN, L.D. An analysis of journal use by structural biologists with applications for journal collection development decisions. College \& Research Libraries, 2001, vol. 62, n 5, p. $422-433$ [en línea]. Disponible en: <http://crl.acrl.org/index.php/crl/article/view/15472> [Consulta: 8 de febrero de 2018].

LEIDING, R. Using citation checking of undergraduate honors thesis bibliographies to evaluate library collections. College \& Research Libraries, 2005, vol. 66, $\mathrm{n}^{\circ} 5$, p. 417-429.

LISÉE, C.; LARIVIÈRE, V. y ARCHAMBAULT, É. Conference proceedings as a source of scientific information: A bibliometric analysis. Journal of the American Society for Information Science and Technology, 2008, vol. 59, $\mathrm{n}^{\circ}$ 11, p. 1776-1784.

MATOS, M.A. Do we have it? A comparative analysis of library journal holdings and works referenced in faculty publications. The Serials Librarian, 2016, vol. 70, n 1-4, p. 260-265.

MATTOS, A.M. y WENSE DIAS, E.J. Desenvolvimento de coleções em bibliotecas universitárias: uma abordagem quantitativa. Perspectivas em Ciência da Informaçao, 2009, vol. 14, no 3, p. 38-60.

MERTON, R.K. Foreword. En: GARFIELD, E. (ed.). Citation indexing: its theory and application in science, technology, and humanities. New Orleans: Wiley, 1979, p. vii-xi [en línea]. Disponible en: $<$ http://www.garfield.library.upenn.edu/cifwd.html $>$ [Consulta: 8 de febrero de 2018].

MILL, D.H. Undergraduate information resource choices. College \& Research Libraries, 2008, vol. 69, n 4, p. 342355.

MILOJEVIĆ, S. How are academic age, productivity and collaboration related to citing behavior of researchers? PloS ONE, 2012, $\quad$ vol. $7, \quad \mathrm{n}^{\mathrm{o}} \quad 11 \quad$ [en $\quad$ línea]. $<$ http://journals.plos.org/plosone/article?id=10.1371/journal.pone.0049176> [Consulta: 8 de febrero de 2018].

MONGEON, P. y PAUL-HUS, A. The journal coverage of bibliometric databases: A comparison of Scopus and Web of Science. Scientometrics, 2016, vol. 106, $\mathrm{n}^{\circ}$ 1, p. 213-228.

NEDERHOF, A.J.; VAN LEEUWEN, T.N. y VAN RAAN, A.F.J. Highly cited non-journal publications in political science, economics and psychology: A first exploration. Scientometrics, 2010, vol. 83, n⿳⺈ 2, p. 363-374.

NOE, J. y FURAY, J. Like a hurricane: A citation analysis of emergency management scholarly literature. Community \& Junior College Libraries, 2013, vol. 19, $\mathrm{n}^{\circ}$ 1-2, p. 21-50.

PINELLI, T.E. The information-seeking habits and practices of engineers. Science \& Technology Libraries, 1991 , vol. $11, \mathrm{n}^{\mathrm{o}} 5$, p. 5-25.

PINELLI, T.E. Distinguishing engineers from scientists - The case for an engineering knowledge community. Science \& Technology Libraries, 2001, vol. 21, no 3-4, p. 131-163.

ROMANOS DE TIRATEL, S. Conducta informativa de los investigadores argentinos en Humanidades y Ciencias Sociales. Revista Española de Documentación Científica, 2000, vol. 23, no 3, p. 267-285 [en línea]. Disponible en: $<$ http://redc.revistas.csic.es/index.php/redc/article/view/324> [Consulta: 8 de febrero de 2018].

ROSENBERG, Z. Citation analysis of M.A. theses and Ph.D. dissertations in sociology and anthropology: An assessment of library resource usage. The Journal of Academic Librarianship, 2015, vol. 41, nº 5, p. 680-688.

RUCINSKI, T.L. The elephant in the room: Toward a definition of grey legal literature. Law Library Journal, 2015, vol. $107, \mathrm{n}^{\circ} 4$, p. 543-559.

SANZ CASADO, E. Manual de estudios de usuarios. Madrid: Fundación Germán Sánchez Ruipérez, 1994.

SMITH, L.C. Citation analysis. Library Trends, 1981, vol. 30, nº 1, p. 83-106.

SMYTH, J.B. Tracking trends: Students' information use in the social sciences and humanities, 1995-2008. Portal: Libraries and the Academy, 2011, vol. 11, n 1, p. 551-573.

SOLÓRZANO ÁLVAREZ, C.E.; MESA FLEITAS, C.M.E.; RODRÍGUEZ SÁNCHEZ, Y. y CAÑEDO ANDALIA, R. Análisis informétrico de la citación en la Revista Cubana de Angiología y Cirugía Vascular en el período 2000- 
2004. ACIMED, 2006, vol. 14, $\mathrm{n}^{\mathrm{o}}$ 5, p. 1 [en línea]. Disponible en: $<$ http://scielo.sld.cu/scielo.php?script=sci_arttext\&pid=S1024-94352006000500013> [Consulta: 8 de febrero de 2018].

STONE, S. Humanities scholars: Information needs and uses. Journal of Documentation, 1982, vol. 38, n 4, p. $292-$ 313.

TANNURI DE OLIVEIRA, F.E.; DE CASTRO SILVA, H. y MOREIRA GARCÍA, R. El uso de la información científica en la producción de tesis de posgrado de la Universidade Estadual Paulista, Marília. Scire, 2007, vol. 13, $\mathrm{n}^{\circ}$ 2, p. 129-137 [en línea]. Disponible en: <http://www.ibersid.eu/ojs/index.php/scire/article/view/1721> [Consulta: 8 de febrero de 2018].

TUCKER, C. Analyzing faculty citations for effective collection management decisions. Library Collections, Acquisition and Technical Services, 2013, vol. 37, n 1-2, p. 19-33.

URBANO SALIDO, C. El análisis de citas en trabajos de investigadores como método para el estudio del uso de información en bibliotecas. Anales de Documentación, 2001, vol. 4, p. 243-266 [en línea]. Disponible en: $<$ http://revistas.um.es/analesdoc/article/view/2281> [Consulta: 8 de febrero de 2018].

VRETTAS, G. y SANDERSON, M. Conferences versus journals in computer science. Journal of the Association for Information Science and Technology, 2015, vol. 66, n 12, p. 2674-2684.

WAINER, J.; PRZIBISCZKI DE OLIVEIRA, H. y ANIDO, R. Patterns of bibliographic references in the ACM published papers. Information Processing \& Management, 2011, vol. 47, nº 1, p. 135-142.

WATKINS, C.A. y GUNAPALA, N. Determining the core literature of water: A citation analysis of faculty from New Mexico State University. Journal of Agricultural \& Food Information, 2013, vol. 14, nº 3, p. 191-208.

ZHANG, L. Citation analysis for collection development: A study of international relations journal literature. Library Collections, Acquisitions, \& Technical Services, 2007, vol. 31, n 3-4, p. 195-207. 